\section{Sicherer Ausschluss durch Wells-Score und D-Dimer-Test}

\author{
Die Verdachtsdiagnose Lungenarterienembolie wird häufig \\ gestellt, aber nur bei 10-15\% der Patienten tatsächlich \\ bestätigt. G.-J.Geersing haben nun die Kombination von \\ Wells-Score und D-Dimer-Test als zuverlässiges diagnostisches \\ Instrument validiert.
} BMJ 2012; 345: e6564

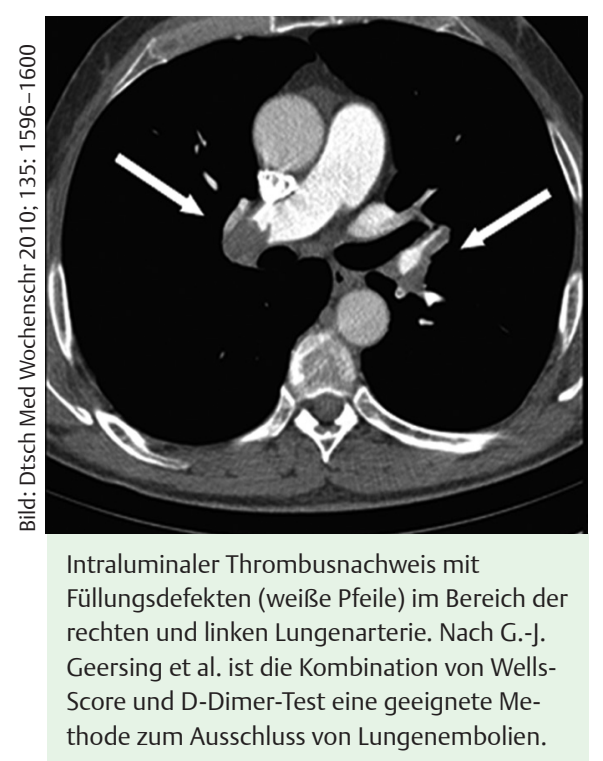

Die prospektive Kohortenstudie (Amsterdam Maastricht Utrecht Study on thrombo-Embolism: AMUSE-2) ergab eine Fehlerrate von unter $2 \%$ und lag damit im Bereich der Spiralcomputertomografie. Mehr als 300 Hausärzte nahmen an der Studie teil. Aufgenommen wurden Patienten, die mit plötzlicher oder plötzlich verschlechterter Dyspnoe, inspiratorischen Brustschmerzen oder ungeklärtem Husten in die Praxis gekommen waren. Ausschlusskriterium war u.a. eine vorbestehende Antikoagulation.

Mit dem Wells-Score werden klinische Anzeichen einer Lungenembolie, Tachykardie, Immobilisation, Tumorerkrankungen, Hämoptysen sowie einer positive Vorgeschichte erfragt und die Wahrscheinlichkeit anderer Diagnosen eingeschätzt. Bei einem Wells-Score $\leq 4$ und einem negativen D-Dimer-Test $(<80 \mathrm{ng} / \mathrm{ml})$

bestand ein niedriges Risiko für eine Lungenarterienembolie. Trotzdem wurden alle Patienten in eine Notfallambulanz überwiesen, wo zusätzlich eine bildgebende Diagnostik erfolgte, und 3 Monate weiter beobachtet.

Die 598 Patienten waren durchschnittlich 48 Jahre alt und zu $71 \%$ Frauen. In 73 Fällen (12,2\%) lag eine Lungenarterienembolie vor. 272 der 598 Patienten wurden der Niedrigrisikogruppe zugeteilt. Bei 4 der 272 Patienten wurde eine Lungenembolie diagnostiziert. Die Fehlerrate lag bei $1,5 \%$ (95\%-Konfidenzintervall [KI] 0,4-3,7\%), die Effizienz dieser Strategie bei 45,5\% (272/598). Die Sensitivität und Spezifität für die Kombination eines Scores $\leq 4$ und negativem D-Dimer-Test betrugen 94,5\% (95\%-KI 86,6-98,5\%) und $51 \%$ (95\%-KI 46,7-55,4\%). Bei einer niedrigeren Schwelle im Wells-Score von $<2$ und einem negativen D-Dimer-Test (168 Patienten, 2 Lungenarterienembolien) lag die Fehlerrate bei 1,2\% (95\%-KI $0,1-4,2 \%$ ). Sensitivität und Spezifität betrugen 97,3\% (95\%-KI 90,5-99,7\%) und $31,6 \%$ (27,7-35,8\%). Die Effizienz dieser Strategie lag bei $28,1 \%(168 / 598)$.

\begin{abstract}
Fazit
Die kombinierte Auswertung von Wells-Score und D-Dimer-Test ist eine schnelle, zuverlässige und zudem kostengünstige Methode zum Ausschluss von Lungenarterienembolien. Nach Meinung der Autoren sollten nach der Validierung nun weitere Studien zur Praktikabilität und ökonomischen Relevanz folgen.
\end{abstract}

\section{Dr. Susanne Krome, Melle}

Tuberkulose

\section{Körper reagiert häufig falsch}

Mykobakterien können sich im Körper ausbreiten, weil das Immunsystem die „falsche" Immunreaktion einsetzt. Zu diesem Ergebnis kommt ein Forscherteam an der University of California. Anstatt die bakterielle Infektion zu bekämpfen, produzieren Patienten mit einer schweren Infektion ein Protein, das Viren angreift. Die im Wissenschaftsmagazin „Science“ veröffentlichten Ergebnisse könnten erklären, warum Viren den Organismus anfälliger für bakterielle Infektionen machen. Ein Höhepunkt der TBC-Infektionen im Frühling könnte daher mit den Auswirkungen der im Winter im Umlauf befindlichen Viren zusammenhängen.

Das Team um Robert Modlin identifizierte dieses Phänomen zuerst bei Lepra. Bei der Untersuchung der Hautverletzungen von Leprapatienten fand man 2 verschiedene Immunproteine. Bei weniger schwer erkrankten Patienten wurde mit Interferon gamma ein Protein festgestellt, das mit einer Immunreaktion auf Bakterien in $\mathrm{Zu}$ sammenhang gebracht wird. Bei schwerer Erkrankten fanden die Experten mit Interferon beta ein Protein, das mit einer Reaktion auf Viren in Verbindung steht. Weitere Untersuchungen zeigten, dass Gene für Interferon beta bei schwereren Erkrankungen häufiger im Blut exprimiert wurden. Laut den Forschern reagiert der Körper bei schweren Erkrankungen so, als würde er einen Virus angreifen. Damit bleiben die Bakterien verborgen und können sich ungehindert in den Zellen verbreiten.

Interferon beta ist jedoch nicht nur im Kampf gegen Bakterien unwirksam, es kann auch die Aktivität von Interferon gamma blockieren. So können die Bakterien nach Angaben der Autoren Fuß fassen. Modlin zufolge legen die Studienergebnisse nahe, dass ein Ansteigen oder Abnehmen eines dieser beiden Proteine die Balance von einer leichten zu einer schwereren Erkrankung verschieben könnte. „Wir könnten Behandlungsmöglichkeiten finden, die bestimmte Interferonreaktionen blockieren oder verstärken und so eine effektive Strategie zum Schutz gegen bakterielle Infektionen entwickeln", so Modlin.

Nach einer Mitteilung von pressetext Nachrichtenagentur $\mathrm{GmbH}$ 


\title{
Standards für die Offenlegung von Interessenkonflikten
}

\author{
Bei der Erstellung von medizinischen Leitlinien können \\ Interessenkonflikte die Empfehlungen in unangemessener \\ Weise beeinflussen. T. Langer et al. haben nun den Umgang mit \\ Interessenkonflikten bei der Leitlinienerstellung analysiert. \\ Dtsch Arztebl Int 2012; 109: 836-842
}

Die Autoren ermittelten hierzu über die Datenbank der Arbeitsgemeinschaft der Wissenschaftlichen Medizinischen Fachgesellschaften (AWMF) alle gültigen S2und S3-Leitlinien aus dem Zeitraum August 2009 - Dezember 2011. Jeweils 2 Personen begutachteten diese unabhängig voneinander bezüglich der Offenlegung von Interessenkonflikten. Für alle Leitlinien, in denen dies der Fall war, dokumentierten wiederum 2 Gutachter unabhängig voneinander die Angaben zum Verfahren und zu den Ergebnissen der Erklärungen der Interessenkonflikte. Neben den Leitlinien berücksichtigten die Autoren diesbezüglich auch Leitlinienreports und Methodenberichte. Erfasst wurden
Vortrags-, Schulungs-, Berater- oder Gutachtertätigkeiten, Drittmittelanträge, Eigentumsverhältnisse, Besitz von Geschäftsanteilen, persönliche Beziehungen zu Vertretungsberechtigten eines Unternehmens der Gesundheitswirtschaft, Mitgliedschaft in Berufsverbänden und Fachgesellschaften sowie die politischen, akademischen und wissenschaftlichen Interessen.

Insgesamt werteten die Autoren 297 Leitlinien aus, von denen sich in 60 (20\%) Angaben zu Interessenkonflikten fanden. 53 Leitlinien (18\%) gaben eine Abfrage von Interessenkonflikten an, veröffentlichten jedoch keine genauen Ergebnisse; 184
Leitlinien(62\%) machten keinerlei Angabe $\mathrm{zu}$ Interessenkonflikten. An den 60 Leitlinien, die Angaben zu Interessenkonflikten machten, waren insgesamt 1606 Personen beteiligt, von denen 1379 solche Angaben machten. Von 227 Personen lagen keine Angaben vor.

Bei 680 Personen (49\%) gab es Hinweise auf einen finanziellen Interessenkonflikt. 522 (38\%) erhielten seitens der Medizinindustrie finanzielle Zuwendungen für Vortrags- und Schulungstätigkeiten, 403 (29\%) berichteten über eine Gutachteroder Beratertätigkeit, 316 (23\%) erhielten Unterstützungen für Forschungsvorhaben. In $86 \%$ der Fälle wurden Interessenkonflikte aufgrund der Zugehörigkeit zu Fachgesellschaften oder Berufsverbänden angegeben. Nach der Einführung der neuen AWMF-Regeln wurden Interessenkonflikte deutlich häufiger angegeben (8\% von 256 Leitlinien vor Einführung vs. 95\% von 41 Leitlinien nach Einführung).

\section{Fazit \\ Auch wenn in den neuen Leitlinien der AWMF seit 2011 Interessenkonflikte of- fengelegt werden, fehlen laut Autoren- team nach wie vor Standards für die Be- wertung.}

Dr. Johannes Weiß, Bad Kissingen

\section{Neue EU-Richtlinie zu Tabakprodukten}

Die EU-Kommission hat sich Ende 2012 auf einen Vorschlag für eine neue Tabakproduktrichtlinie geeinigt. Der Entwurf sieht u. a. das Verbot bestimmter Zusatzstoffe in Tabakwaren vor. Darüber hinaus werden Regelungen zu kombinierten und größeren Bildwarnhinweisen sowie zu Verpackungs- und Standardgrößen von Zigaretten vorgeschlagen.

Dazu erklärt die Drogenbeauftragte der Bundesregierung, Mechthild Dyckmans: „Als Drogenbeauftragte begrüße ich die Überarbeitung der Tabakproduktrichtlinie der Europäischen Union. Ein Verbot von suchtfördernden Zusatzstoffen und auffällige Warnhinweise sind aus meiner Sicht geeignete Maßnahmen, damit künftig weniger Menschen zur Zigarette greifen. Dabei gilt es, insbesondere den frühen
Einstieg in das Rauchen bei Kindern und Jugendlichen $\mathrm{zu}$ verhindern. Positiv ist auch, dass die E-Zigarette europaweit einbezogen werden soll.“

Der Vorschlag für eine neue Tabakproduktrichtlinie sieht Regelungen vor zu:

\section{Zusatzstoffen}

- Verbot von Zigaretten mit bestimmten Aromen („characterising flavour")

- Verbot von Tabakprodukten mit Zusätzen, die den Eindruck eines Gesundheitsvorteils verschaffen (z.B. Vitamine) oder belebend wirken (z. B. Koffein).

\footnotetext{
Verpackung und Etikettierung

- Auf den Seiten der Zigarettenverpackung soll der Warnhinweis „Smoking
}

kills - quit now" und der Informationshinweis „Tobacco smoke contains over 70 substances known to cause cancer" in deutscher Sprache aufgedruckt sein. Die Hinweise sollen 50\% der Fläche ausmachen.

- Die Vorder- und Rückseite sollen jeweils mit einem kombinierten BildText-Warnhinweis versehen werden. Dieser soll 75\% der Fläche ausmachen.

- Zigaretten mit einem Durchmesser von unter 7,5 mm gelten aus Gründen des Verbraucherschutzes als irreführend und sind damit verboten.

- Einführung eines Rückverfolgungssystems für jede Zigarettenpackung („Tracking and Tracing") zur Reduzierung des Schwarzmarkts von Zigaretten.

Nach einer Mitteilung der Drogenbeauftragten der Bundesregierung, Berlin 


\section{Erkrankungsrisiko steigt mit Alter und Tabakkonsum}

\author{
Es ist bereits bekannt, dass Rauchen ein starker Risikofaktor \\ für eine chronische Bronchitis ist. H. Meteran et al. \\ haben nun in Dänemark diesen Zusammenhang an einer \\ großen Zwillingskohorte näher untersucht. \\ Lung 2012; 190: 557-561
}

Die Studienpopulation bestand aus zwischen 1931 und 1952 geborenen Zwillingen, die in einem landesweiten dänischen Zwillingsregister eingetragen waren. Im Jahr 2002 nahmen sie an einer Fragebogenstudie zu Gesundheit und Lebensstil teil. Dabei wurden auch Daten zum Nikotinkonsum erfasst. Eine chronische Bronchitis war definiert als eine bejahende Antwort auf die Frage: Hatten Sie während der letzten 2 Jahre an mindestens 3 Monaten pro Jahr Husten mit Auswurf? Aus der ursprünglichen Kohorte von 13649 Zwillingen waren 1146 Zwillingspaare bezüglich ihres Nikotinkonsums diskordant.

Das Durchschnittsalter der Teilnehmer lag bei 58,8 Jahren, 52,7\% von ihnen waren Frauen. Die Prävalenz von gegenwärtigen Rauchern betrug 33\%, die von ehemaligen Rauchern 30\%. 9,7\% der Teilnehmer litten an einer chronischen Bronchitis. Die Prävalenz einer chronischen Bronchitis betrug $4 \%$ bei Teilnehmern, die niemals geraucht hatten, $6,1 \%$ bei ehemaligen Rauchern und 15,9\% bei aktuellen Rauchern. Raucher wurden anhand ihres täglichen Zigarettenkonsums in 3 Gruppen unterteilt (1-10, 11-20 und >20 Zigaretten pro Tag). Dabei stieg das Risiko für eine chro-

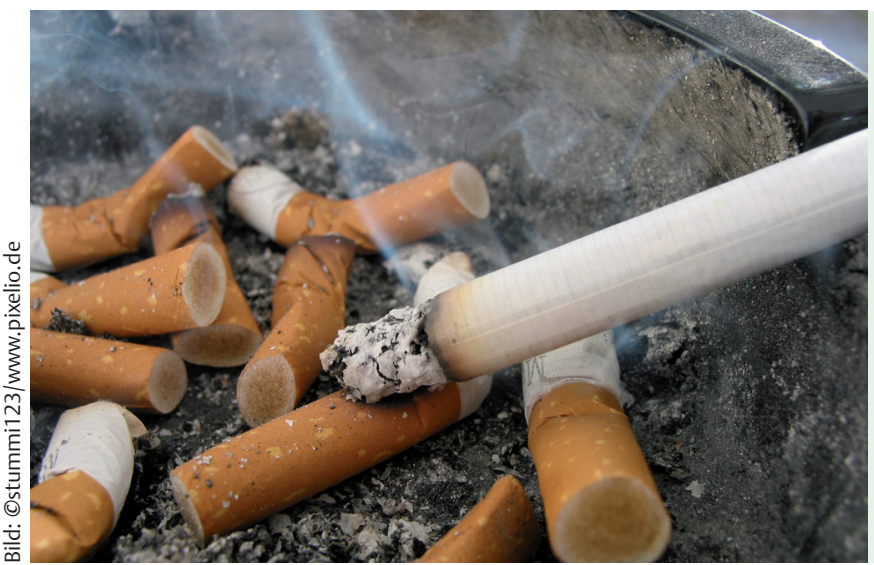
schen Bronchitis umso
Laut Studie steigt das Risiko einer chroniälter ein Mensch ist und umso mehr und länger er raucht.

Onkologie

\section{Frauen sterben häufiger an Lungenkrebs}

Der Lungenkrebs ist auf dem Weg, Brustkrebs als häufigste Krebstodesursache bei Frauen zu überholen, wie ein Forscherteam der Universita degli Studi di Milano ermittelt hat. Dieser Anstieg spiegelt die Zunahme der Frauen wider, die in den 1960er- und 1970er-Jahren begonnen haben zu rauchen. Die Todesfälle durch Lungenkrebs werden laut Carlo La Vecchia in den nächsten Jahren zunächst ansteigen.

Bronchitis dosisabhängig auf gefährte und Rauchen waren unabhängige Prädiktoren einer chronischen Bronchitis mit einem bis zu 10-fach erhöhten Risiko bei den stärksten Rauchern.

Von den 1146 bezüglich ihres Nikotinkonsums diskordanten Zwillingspaaren stieg das Risiko für eine chronische Bronchitis dosisabhängig an, wenn gegenwärtige Raucher mit Teilnehmern, die niemals geraucht hatten, verglichen wurden: Odds Ratio zwischen 1,5 (1-10 Zigaretten) und 3,2 (>20 Zigaretten). Zwischen eineiigen und zweieiigen Zwillingen bestand hierbei kein Unterschied.

\section{Fazit}

Das Risiko für eine chronische Bronchitis steigt mit zunehmendem Alter und Tabakkonsum an. Die Ergebnisse sprechen für eine direkte Beziehung zwischen Rauchen und der Erkrankung, aber auch Umweltfaktoren wie die Exposition gegenüber Passivrauch in der Kindheit spielen eine Rolle, so die Autoren.

\section{Dr. Johannes Weiß, Bad Kissingen}

\section{Über 82000 Todesfälle pro Jahr $\nabla$}

Im Jahr 2013 werden in der EU nach Angaben der Wissenschaftler schätzungsweise 82640 Frauen an Lungenkrebs sterben und 88886 an Brustkrebs. 2015 wird sich dieses Verhältnis geändert haben und mehr Patientinnen werden an Lungenkrebs sterben. Die Forscher analysierten die Krebserkrankungen in der gesamten EU. Details der Studie wurden in der Fachzeitschrift „Annals of Oncology“ veröffentlicht. Die Autoren gingen dabei von den 27 Mitgliedstaaten von 2007 aus und untersuchten 6 Länder im Einzeln. Dazu gehörten Frankreich, Deutschland, Italien, Polen, Spanien und Großbritannien. Analysiert wurden alle Krebsarten mit dem Schwerpunkt auf Magen-, Darm-, Bauchspeicheldrüsen-, Lungen-, Prostata-, Brust- und Gebärmutterkrebs sowie Leukämie.

\section{Trend wird sich wieder umkehren $\nabla$}

Die Zahlen zeigen, dass, obwohl immer mehr Menschen an Krebs erkranken, insgesamt weniger an den Folgen sterben. Verantwortlich für die Zunahme ist die höhere Lebenserwartung. Trotz der Abnahme der Anzahl tödlich verlaufender Erkrankungen, steigt die Todesrate durch Lungenkrebs bei Frauen in Europa. Beim Lungenkrebs erwarten die Studienautoren einen Rückgang der Erkrankungen um das Jahr 2020 oder 2025, da die neue Generation von Frauen seltener raucht.

Nach einer Mitteilung von pressetext Nachrichtenagentur $\mathrm{GmbH}$ 


\title{
Copeptin als Biomarker für Hochrisikopatienten
}

\author{
Zur Mortalitätsprognose bei ambulant erworbener Pneumonie \\ (AEP) werden die Risiko-Scores CRB-65 (Confusion, Respiratory \\ Rate, Blood Pressure, Age $\geq 65$ ) und PSI (Pneumonia Severity \\ Index) häufig empfohlen. M. Kolditz et al. haben überprüft, ob \\ sich mit den Biomarkern Copeptin und MR-proADM (mittregio- \\ nales Proadrenomedullin) die Vorhersage verbessern lässt. \\ Respir Medicine 2012; 106: 1320-1328
}

Die prospektive Untersuchung des prädiktiven Werts der neuen Biomarker wurde mit Entzündungsindikatoren, den genannten Scores sowie dem Score der American Thoracic Society (ATS) und Infectious Diseases Society of America (IDSA) mit 9 Minor-Kriterien verglichen. Dafür standen 51 konsekutiv stationär aufgenommene Patienten mit AEP zur Verfügung. Zu den Ausschlusskriterien gehörten u. a. nosokomiale Pneumonien und Aufnahme auf die Intensivstation innerhalb der ersten 24 Stunden. Die Krankheitsschwere wurde anhand aller Scores beurteilt. Am Morgen nach der stationären Aufnahme wurden Blutproben entnommen. Sämtliche Labormessungen fanden verblindet statt. Die vorher definierten Ergebnisparameter waren Aufnahme auf die Intensivstation oder Mortalität am 7. Tag nach Krankenhausaufnahme (I) sowie klinische Instabilität 72 Stunden nach Blutentnahme (II).

\section{Verbesserte Prädiktion mit Copeptin \\ $\nabla$}

Der Copeptin-Wert erhöhte sich bei Patienten, die in Bezug auf beide Ergebnisparameter schlechte Verläufe hatten. Damit war Copeptin neben dem PSI-Score und den ATS/IDSA-Minor-Kriterien der einzige Parameter, der deutliche Unterschiede bei Patienten mit frühen Zustandsverschlechterungen ergab. Die diagnostische Kapazität von Copeptin, Ereignisse entsprechend (I) vorherzusagen, erreichte einen AUCWert von 0,81 mit einem optimalen Cutoff-Wert von 35 pmol/l. Die Sensitivität betrug $78 \%$, die Spezifität $79 \%$, der positive prädiktive Wert (PPV) 40\% und der negative prädiktive Wert (NPV) $92 \%$. Die Werte für die Vorhersage klinischer Instabilitäten innerhalb von 36 Stunden waren für Copeptin: AUC 0,74, optimaler Cut-off 25 pmol/l, Sensitivität $60 \%$, Spezifität $81 \%$, PPV 75\% und NPV 68\%. Der Biomarker MR-proADM erreichte AUC-Werte von 0,67 für (I) und 0,61 für (II).

Die Anwendung des PSI-Scores für die Vorhersage entsprechend des ersten Ergebnisparameters ergab einen AUC-Wert von 0,75 und war damit Copeptin nicht unterlegen $(p=0,36)$. In der Kombination mit Copeptin stieg dieser Wert auf 0,83. Dagegen schnitt der CRB-65-Score deutlich schlechter ab als der Biomarker (AUC 0,57, $p=0,018)$. Mit der Kombination CRB-65-Score plus Copeptin kam der AUC-Wert nicht an den von Copeptin allein heran (AUC 0,78). Die Vorhersage von Aufnahmen auf die Intensivstation oder Mortalität am 7. Tag nach Krankenhausaufnahme mit 9 ATS/IDSA-Minor-Kriterien war ebenso zuverlässig wie mit Copeptin (AUC 0,81). Die Kombination aus den Kriterien plus Biomarker verbesserte den AUC-Wert auf 0,85. Bei einem optimalen Cut-off bei mehr als 2 Kriterien lag die kombinierte Sensitivität bei $89 \%$ und die Spezifität bei $67 \%$.

\section{Fazit}

Copeptin ermöglichte bei Patienten mit AEP in dieser Studie eine bessere Prädiktion früher Zustandsverschlechterungen und anhaltender klinischer Instabilitäten. Zusätzlich verbesserte der Biomarker die prädiktiven Eigenschaften etablierter klinischer Scores, so die Autoren.

\section{Matthias Manych, Berlin}

\section{Neue Studie zu Smog in China}

Die Ostküste Chinas gehört zu den Regionen der Erde mit der größten Luftverschmutzung durch reaktive Stickstoffverbindungen. Diese Entwicklung hat sich in den vergangenen 30 Jahren erheblich verschlimmert. Ohne Gegenmaßnahmen werde sich das Problem weiter verschärfen, warnt eine deutsch-chinesische Studie in der aktuellen Ausgabe der Fachzeitschrift „Nature“. Unter den Autoren sind Ökologen und Agrarwissenschaftler des deutsch-chinesischen Graduiertenkollegs an der Universität Hohenheim und der China Agricultural University in Peking.

Die starke Luftverschmutzung ist die Kehrseite der boomenden Wirtschaft: Kohle ist die wichtigste Energiequelle, die Zahl der Autos wächst exponentiell, und in der Landwirtschaft haben sich sowohl der Tierbestand als auch die Düngermenge auf den Feldern in den vergangenen 30 Jahre verdreifacht. Entsprechend stark ist die Luftbelastung angewachsen. Ein weiterer Trend wird aus den Analysen sichtbar: die Belastung mit reduzierten Stickstoffverbindungen überwiegt zwar, aber der Anteil an oxidierten Stickstoffverbingungen hat noch stärker zugenommen. „Das heißt, die Belastung aus Verkehr und Industrie steigt noch deutlicher, als die aus der Landwirtschaft", erklärt Prof. Andreas Fangmeier von der Universität Hohenheim.

Die Autoren der Studie zeigen, wie sich die Luftverschmutzung und die Belastung der Umwelt durch Stickstoffverbindungen zwischen 1980 und 2010 immer weiter verschärft haben: „Kaum irgendwo anders auf der Welt ist die Stickstoffbelastung so hoch wie im Osten Chinas“, so Prof. Fangmeier. „Das Reich der Mitte hat heute dieselben Probleme wie die am stärksten betroffenen Regionen Europas vor 20 bis 30 Jahren. “ Die Folgen: extrem hohe Belastungen durch Feinstaub mit entsprechenden gesundheitlichen Gefahren und Stickstoffeinträge in die Ökosysteme in einer Höhe, die weit über den tolerierbaren Schwellenwerten liegt. Ohne Gegenmaßnahmen werde sich das Problem in Zukunft noch weiter verschärfen, warnen die Forscher.

Nach einer Mitteilung der Universität Hohenheim, Stuttgart 


\section{First-Line-Chemotherapie häufiger als erwartet}

\author{
Studien haben gezeigt, dass ältere Patienten mit fortgeschritte- \\ nem nicht kleinzelligem Lungenkarzinom (NSCLC) relativ selten \\ eine First-Line-Chemotherapie erhalten. Über die Therapie bei \\ jüngeren Patienten war bisher wenig bekannt. D. P. Ritzwoller et \\ al. haben nun herausgefunden, dass sie häufiger zur primären \\ Behandlung von NSCLC angewandt wird als angenommen. \\ Lung Cancer 2012; 78: 245-252
}

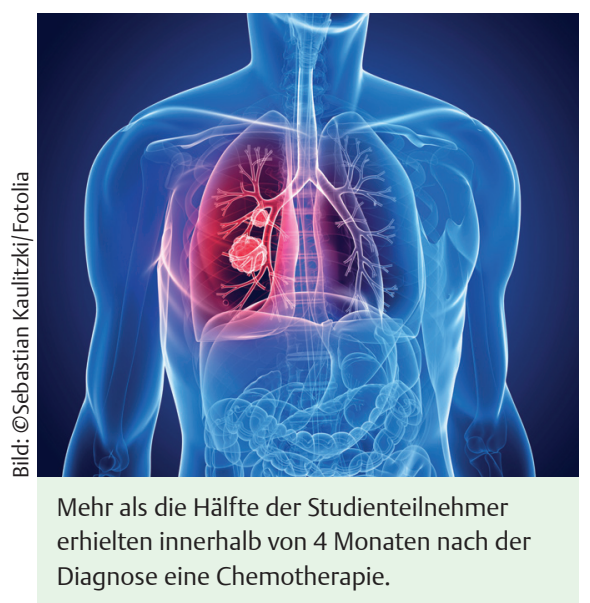

Bei der Behandlung von fortgeschrittenem NSCLC spielen in den USA das Verhalten von Gesundheitsorganisationen (Health Maintanance Organizations: HMO), die Abwägung hoher Kosten und der Einsatz neuer Therapeutika, wie Bevacizumab und Erlotinib eine Rolle. Diese Faktoren führen zu Variationen in der Therapie von NSCLC, die Ritzwoller et al. näher untersuchten. Dazu identifizierten sie 6614 Patienten mit NSCLC im Stadium IIIB/IV im Alter über 21 Jahre in $4 \mathrm{HMO}$, die alle beim amerikanischen Cancer Research Network (CRN) mitwirkten. Sie nahmen demografische Daten und Angaben zu Begleiterkrankungen, Tumorcharakteristika und chemotherapeutische Behandlung in logistische Regressionsmodelle auf, um die mit der Einnahme der Chemotherapeutika assoziierten Faktoren zu identifizieren.

Wie sich anhand der Auswertungen herausstellte, erhielten 3612 (55\%) Patienten innerhalb von 120 Tagen nach der Diagnose eine Chemotherapie. Der Anteil stieg vom Jahr 2000 bis 2007 von 52\% auf 59\% jährlich an ( $p=0,001)$. Der Erhalt von Chemotherapeutika lag bei Patienten im Alter $<65$ Jahre mit 64\% deutlich höher als bei Patienten $\geq 65$ Jahre mit $46 \%$ und stand in inverser Relation zu den Stadien und Komorbiditäten. Am häufigsten erhielten die Patienten Carboplatin und Paclitaxel (zusammen 55,7\% bei Patienten <65 Jahren bzw. $51,1 \%$ bei denen ab 65 Jahren). Der Einsatz von Erlotinib (2,3\% unter 65 Jahren bzw. 2,4\% ab 65 Jahren) und Bevacizumab (kombiniert mit Carboplatin und Paclitaxel 4,4\% unter 65 Jahren bzw. 1,7\% ab 65 Jahren) in den späteren Jahren der Beobachtungszeit war mit einer Veränderung in der Verteilung der First-Line-Chemotherapien assoziiert. So war Erlotinib ab 2005 die am häufigsten eingesetzte Einzelsubstanz. Noch auffälliger war der Anstieg des Einsatzes der Dreifachkombination von Bevacizumab-Carboplatin-Paclitaxel, die ab 2007 11\% aller First-Line-Behandlungen ausmachte. Bei Patienten, die 30 Tage nach der Diagnose noch lebten, lag die primäre Anwendung von Chemotherapie in der älteren Population ab 65 Jahren höher, als bisher angenommen. Noch höher lag der First-Line-Einsatz bei jüngeren Patienten bis 65 Jahre. Gleichzeitig nahm die Anwendung von Chemotherapeutika im Verlauf der Beobachtungszeit zu.

\section{Fazit}

Künftige Studien könnten nach Ansicht der Autoren die im CRN verfügbaren HMO-Daten über die Primärtherapie nutzen, um chemotherapiespezifische, vergleichende Effektivitätsstudie durchzuführen, welche die vorliegenden Studien ergänzen und ausweiten.

Dr. Volker Kriegeskorte, Buchloe
Epigenetik

\section{Rauchende Omas vererben Asthma}

Großmutters Zigarettenkonsum könnte die Ursache für die Asthma-Erkrankung der Enkel sein, wie das Biomedical Research Institute in Los Angeles anhand einer Studie feststellte. Anhand von Ratten hat man den Zusammenhang von Nikotinkonsum und Asthma entdeckt. Demnach kann Nikotin eine epigenetische Markierung auf dem Genom hinterlassen, das die Nachkommen anfälliger für Atemwegserkrankungen macht.

\section{Vermeidbares Risiko \\ $\nabla$}

Wenn die Großmutter sogar während ihrer Schwangerschaft geraucht hat, ist das Risiko, dass ihre Enkel an Asthma erkranken, noch höher. Der Zigarettenkonsum beeinträchtigt also nicht nur die Raucherin selbst oder ihre Kinder, sondern sogar noch die kommende Enkelgeneration. „Asthma ist die häufigste chronische Erkrankung im Kindesalter, was zu einer deutlichen Auswirkung auf das Leben von Kindern führt und zudem die Kosten für die medizinische Versorgung aller hochschraubt", sagt Virender Rehan, einer der Studienleiter. „Auch wenn viele Umweltfaktoren zum Asthma beitragen, ist das Rauchen in der Schwangerschaft ein vermeidbares Risiko. Würden Frauen während der Schwangerschaft nicht rauchen, könnte sich das auf die Gesundheit der nächsten Generationen auswirken", erklärt John Torday.

\section{Experten bestätigen Annahmen $\nabla$}

Dass die Rauchgewohnheiten der Oma die Gesundheit der Enkelgeneration beeinträchtigen, erscheint Erika von Mutius, Medizinerin an der Haunerschen Kinderklinik der Ludwig-Maximilian-Universität in München plausibel. „Das kann in der Tat möglich sein. Es gab eine frühere Studie an Kindern mit einer derartigen Beobachtung", sagt von Mutius gegenüber pressetext. Man könnte sich das durch epigenetische Mechanismen durchaus vorstellen.

Nach einer Mitteilung von pressetext Nachrichtenagentur GmbH 


\title{
Nutzen von Azithromycin bei Bronchialveränderungen
}

\author{
Die Rate an Exazerbationen bei Bronchiektasen wird mit \\ 1,5-6,5 pro Patient und Jahr angegeben. Für deren Prävention \\ und Behandlung stehen nur wenige evidenzbasierte Therapien \\ zur Verfügung. C. Wong et al. haben nun den Nutzen des \\ Makrolidantibiotikums Azithromycin bei Patienten mit nicht \\ mukoviszidosebedingten Bronchiektasen untersucht. \\ Lancet 2012; 380: 660-667
}

An der randomisierten, doppelblinden EMBRACE-Studie (Effectiveness of Macrolides in Patients with Bronchiectasis using Azithromycin to control Exacerbations) nahmen Patienten teil, die folgende Kriterien erfüllten: Alter $\geq 18$ Jahre, mindestens eine behandlungsbedürftige Exazerbation im vergangenen Jahr und eine CTbestätigte Brochiektase-Diagnose. Die Patienten erhielten entweder $500 \mathrm{mg}$ Azithromycin oder Placebo an 3 Tagen in der Woche über insgesamt 6 Monate. Es folgte ein Follow-up von weiteren 6 Monaten. Untersuchungen fanden nach 4, 13, 26, 39 und 52 Wochen statt; außerdem wurden die Patienten zwischen diesen Terminen telefonisch befragt. Neben der Spirometrie beurteilten die Mediziner auch Daten aus dem St. George's Respiratory Questionnaire (SGRQ), dem 6-Minuten-Gehtests, Messungen des C-reaktiven Proteins (CRP) und zu Nebenwirkungen. Die Endpunkte dieser Studie waren ereignisbasierte Exazerbationen innerhalb der ersten 6 Monate, $\mathrm{FEV}_{1}$ und SGRQ-Score am Therapieende.

\section{Weniger Exazerbationen}

Insgesamt konnten 71 Teilnehmer in die Azithromycin- und 70 in die Placebogruppe randomisiert zugeordnet werden. Die Adhärenz betrug im Verum- 97,9\% und im Placeboarm 98,3\%.Während es unter dem Antibiotikum nach 6 Monaten zu 42 Exazerbationen kam, traten unter Placebo 103 Ereignisse auf. Daraus ergaben sich ereignisbasierte Exazerbationsraten von 0,59 (Azithromycin) und 1,57 (Placebo) mit einer relativen Reduktion von 62\% $(p<0,0001)$. Die entsprechenden Ergebnisse nach 12 Monaten waren: 109 Exazerbationen (Azithromycin), 178 Exazer- bationen (Placebo), Exazerbationsraten 1,58 bzw. 2,73, relative Reduktion zugunsten von Azithromycin 42\% ( $p<0,0001)$. Da $31 \%$ der Verum- und $66 \%$ der Placebopatienten in 6 Monaten mindestens 1 ereignisbasierte Exazerbation erlitten, erreichte die Risikoreduktion $52 \%(\mathrm{p}<0,0001)$; nach 12 Monate waren es $25 \%(0,005)$. Die Zeit, in der es bei mindestens $25 \%$ der Patienten nach 6 Monaten zur ersten Exazerbation kam, lag im Verum- bei 104, im Placeboarm bei 21 Tagen $(p<0,0001)$. Nach 12 Monaten betrugen die Zeiträume 239 vs. 85 Tage $(\mathrm{p}<0,0001)$.

Weder die symptombasierten Exazerbationen noch die $\mathrm{FEV}_{1}$-Veränderungen (Prä-, Post-Bronchodilatation) ergaben deutliche Unterschiede zwischen den Studienarmen. Gleiches galt auch für den SGRQGesamtscore. Die CRP-Konzentrationen, die nur nach 6 Monaten gemessen wurden, gingen im Vergleich zu den Ausgangswerten unter Azithromycin zurück und stiegen in der Placebogruppe an $(-13,8$ vs. $40,1 \%, p=0,006)$. Die Verumtherapie wurde allgemein gut vertragen und verursachte überwiegend leichte gastrointestinale Nebenwirkungen.

\section{Fazit}

Patienten mit nicht mukoviszidosebedingten Bronchiektasen hatten in der Studie mit der Azithromycin-Therapie weniger Exazerbationen. Bis zur ersten Verschlimmerung verging eine längere Zeit. Dieser Nutzen hielt 6 Monate nach Behandlungsende an. Die Autoren empfehlen eine sorgfältige Patientenauswahl und weisen auf das Risiko von Macrolidresistenzen hin.

Matthias Manych, Berlin
Rauchen

\section{Rauchausstieg auch im Alter effektiv}

Raucher steigern mit jeder Zigarette ihr Herzinfarkt- und Schlaganfall-Risiko. Doch auch umgekehrt gilt: Selbst wer erst im fortgeschrittenen Alter mit dem Rauchen aufhört, senkt sein Risiko bereits innerhalb kürzester Zeit nach dem Ausstieg erheblich. Das fanden Forscher des Deutschen Krebsforschungszentrums (DKFZ) jetzt anhand einer Studie an Einwohnern aus dem Saarland heraus.

Für ihre Studie analysierten Prof. Hermann Brenner und Kollegen die Daten von 8807 Personen im Alter von 50-74 Jahren. „Wir konnten zeigen, dass Raucher ein mehr als doppelt so hohes Risiko für Herz-Kreislauf-Erkrankungen haben wie Nichtraucher. Ehemalige Raucher sind dagegen fast genauso selten betroffen wie Menschen gleichen Alters, die nie geraucht haben“, sagt Brenner. „Außerdem erkranken Raucher deutlich früher als Personen, die nicht oder nicht mehr rauchen." So hat beispielsweise ein 60 -jähriger Raucher das Herzinfarkt-Risiko eines 79-jährigen Nichtrauchers und das Schlaganfall-Risiko eines 69-jährigen Nichtrauchers. Dabei wirken sich die Tabakdosis und die Dauer des Konsums auf das Erkrankungsrisiko aus: Je mehr Zigaretten pro Tag über einen längeren Zeitraum geraucht werden, desto höher ist das Risiko.

Der positive Effekt eines Rauchausstiegs macht sich bei den Studienteilnehmern bereits nach kurzer Zeit bemerkbar. „Verglichen mit Personen, die weiterhin rauchen, ist das Risiko für einen Herzinfarkt und für einen Schlaganfall bereits während der ersten 5 Jahre nach der letzten Zigarette mehr als $40 \%$ niedriger“, betont Carolin Gellert, die Erstautorin der Studie, die Anfang $2013 \mathrm{im}$ „European Journal of Epidemiology“ veröffentlicht wurde. Die Ergebnisse legen nahe, dass Programme zur Tabakentwöhnung, die sich bislang auf jüngere Teilnehmer konzentrieren, auf ältere Personen ausgeweitet werden sollten.

Nach einer Mitteilung des DKFZ, Heidelberg 